\title{
The management of bulimia nervosa and other binge eating problems
}

\author{
Christopher G. Fairburn
}

This article is about the management of eating disorders in which binge eating is a prominent feature. These disorders include bulimia nervosa, the most common eating disorder, and 'binge eating disorder', a provisional new diagnosis included in DSM-IV. In addition, binge eating is seen in anorexia nervosa and in many atypical eating disorders.

Until recently, there was confusion over what constitutes a 'binge'. In general usage the term does not have a truly specific meaning; indeed, over the years dictionary definitions have changed. TheOxford English Dictionary traces the use of 'binge' back to the mid-19th century when it referred to "a heavy drinking bout, hence a spree", and this remains one of its meanings today. Only more recently has the word been applied to eating. Nowadays dictionaries refer to overeating, and the term 'indulgence' may be used. However, these definitions neglect a central characteristic of binge eating, as seen in people with eating disorders; namely, the accompanying sense of loss of control. It is this loss of control over eating that distinguishes binge eating from everyday overeating and gluttony.

Technically, binges (or bulimic episodes) have two essential features: first, a large amount of food is eaten; and second, there is an associated sense of loss of control. Hence the DSM-IV definition:

"An episode of binge-eating is characterized by both of the following:

(1) eating, in a discrete period of time (e.g. within any two-hour period), an amount of food that is definitely larger than most people would eat during a similar period of time and under similar circumstances; and

(2) a sense of lack of control over eating during the episode (e.g. a feeling that one cannot stop eating or control what or how much one is eating)."
It is important to note that the evaluation of the amount eaten is contextual; that is, account is taken of what would be the usual quantity to eat under the circumstances. In clinical practice some patients are encountered whose binges involve the consumption of modest amounts of food, this being especially true of patients with anorexia nervosa. Such episodes do not fulfil the technical definition of a binge, although they may seem very similar. Sometimes they are referred to as 'subjective bulimic episodes' or 'subjective binges'. Table 1 shows the binge eating of a patient with bulimia nervosa.

\section{Diagnostic characteristics of bulimia nervosa, binge eating disorder and anorexia nervosa}

Figure 1 shows how binge eating problems are classified. The diagnostic criteria for the relevant eating disorders are described below.

\section{Bulimia nervosa}

Three features have to be present for someone to be said to have bulimia nervosa:

(1) The person must have frequent binges; that is, he or she must consume genuinely large amounts of food, taking into account the context in which the food is eaten, and the person must have a sense of loss of control at the time.

(2) The person must regularly use one of a variety of extreme measures for controlling shape or weight. These measures include self-induced vomiting, misusing laxatives or diuretics, overexercising and intense dieting or fasting.

(3) The person must be excessively concerned about his or her shape or weight or both. There should be an intense fear of fatness or weight gain, and

Christopher G. Fairburn, FRCPsych, is Wellcome Principal Research Fellow and Professor of Psychiatry in the Department of Psychiatry at the University of Oxford. He is well known for his research on eating disorders and their treatment. He recently edited (with K. D. Brownell) Eating Disorders and Obesity: A Comprehensive Handbook, and he has written a book for sufferers and their families, entitled Overcoming Binge Eating. Both books were published in 1995 by Guilford Press, New York. 


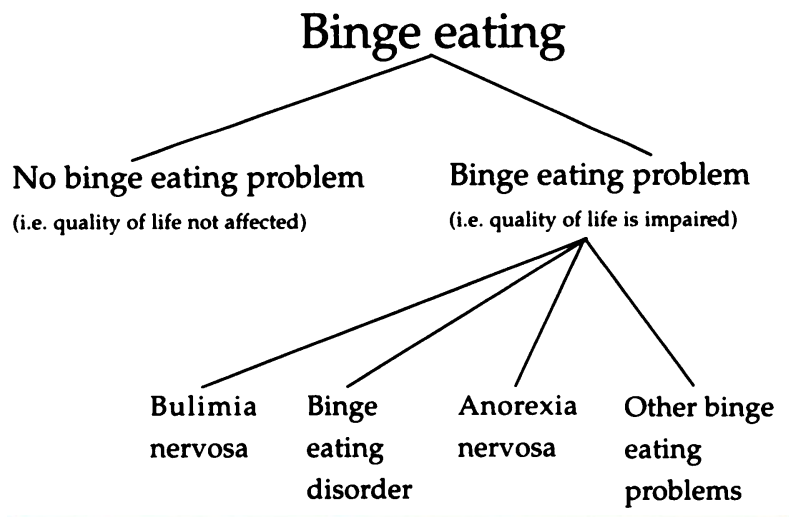

Fig. 1. The classification of binge eating. Adapted with permission from Fairburn, 1995.

self-worth should be judged largely or even exclusively in terms of shape or weight.

There is one other requirement; the person should not have anorexia nervosa. In effect, this means that the person should not be significantly underweight. In practice, the great majority of people with bulimia nervosa have a weight that is in the normal range.

\section{Binge eating disorder}

People with binge eating disorder have repeated binges in the absence of the extreme measures to control shape and weight that characterise bulimia nervosa (item 2 above). In the past they were sometimes called 'compulsive overeaters'. Among patient samples, many such people are overweight.

\section{Anorexia nervosa}

Three features must be present for someone to be said to have anorexia nervosa:

(1) The person should be significantly underweight, and this should be the result of his or her own efforts. A widely used weight threshold is being more than $15 \%$ below the expected weight for the person's age, sex and height.

(2) (Females only.) Should not be menstruating (unless she is taking an oral contraceptive).

(3) The person should be highly concerned about his or her shape or weight or both. However, rather than worrying about being underweight, the person should be afraid of gaining weight. As in bulimia nervosa, self-worth should be judged largely or even exclusively in terms of shape or weight. People with anorexia nervosa are sometimes said to have a morbid fear of fatness and their dieting may be said to be driven by a relentless pursuit of thinness. These attitudes to shape and weight are similar to those found in bulimia nervosa.

\section{Table 1. Binge eating of a patient with bulimia nervosa: illustration of a food monitoring record that would be} completed by the patient. B, binge; V, vomit; L, laxative. Adapted with permission from Fairburn, 1995

\begin{tabular}{|c|c|c|c|c|c|}
\hline Time & Food and liquid consumption & Place & B & $\mathrm{V} / \mathrm{L}$ & Comments \\
\hline \multirow[t]{2}{*}{7.35} & 1 onapefruct & Kadien & & & Fed really fat \\
\hline & 1 cup llack ooffee & Kadien & & & \\
\hline 11.10 & Tapple & wate & & & \\
\hline 3.15 & 27 wix., 1 Gread rode & Highst. & * & & \\
\hline \multirow[t]{2}{*}{3.30} & 1 frucutcalbe, 2 chocolate egge & Matare & * & & $\begin{array}{l}\text { Euryoure loaked at me in the } \\
\text { market. I' } m \text { out of control. }\end{array}$ \\
\hline & 2 bread rolls, punt mille & Kathen & * & $v$ & Thate meswelf. \\
\hline \multirow[t]{2}{*}{5.10} & $\begin{array}{l}2 \text { bouls coreal, } 1 \text { puta bread } \\
\text { with cottage cheese }\end{array}$ & Kadien & * & & Ican t tstape orying. \\
\hline & 1 glase water & Kathen & & & \\
\hline 6.00 & 1 Gaked potato, 1 can Tabe & Vanocaside & * & $v$ & \\
\hline 9.00 & 1 cup Stimline soup, ice cube & Katren & & & Weighed meswelf: 9st 8cb-taa \\
\hline 9.20 & 1 aup coffee & Kudien & & & heavy. Feel fat and ugly. \\
\hline 1000 & 1 coffee (telack) & Stating noom & & & \\
\hline \multirow[t]{6}{*}{1120} & 1 cosfee (alack) & Kadren & * & & Why da? da thice? I wavet to be \\
\hline & 6shondread biscutats & & * & $v$ & then. \\
\hline & 4 pieces of chacalate & & * & $v$ & Teanit help at. \\
\hline & 2 picees of toast & & & & \\
\hline & 2 glasses of unater & & & & Weighed 9at 7t6-fat. \\
\hline & & & & 2 & Took 24 Neplax. \\
\hline
\end{tabular}




\section{The scale of the problem}

Since 1980, there have been numerous studies of the prevalence of binge eating (for review see Fairburn \& Beglin, 1990). These studies have mainly focused on women aged between 14 and 40 years, as they are thought to be most at risk, and the majority have collected their data by asking subjects to complete simple pencil-and-paper questionnaires. They have found that many young women report "binge eating". Indeed, across the studies, over one-third do so and about $15 \%$ report that they binge at least weekly. For a number of reasons, these figures must be questioned. One is that most studies have not used the formal definition of a binge (described above). Instead, many have simply asked questions like "Do you binge?" and have then taken subjects' responses more or less at face value. As a result, the figures are more likely to reflect those for any form of perceived overeating, rather than true binge eating. Another problem is that over one-half of the studies have focused on female students and often those enrolled at prestigious American universities. Such subjects are unlikely to be representative of young women in general, who may have different rates of binge eating.

What is needed are studies in which representative general population samples are recruited and assessed by standardised interview, since such interviews are regarded as the best way of assessing binge eating (see Wilson, 1993). There has been just one study of this type (based in Oxfordshire) and it found that among women aged between 16 and 35 years, $10 \%$ had at least monthly binges and 3\% were binge eating at least weekly. These figures are much lower than those obtained using self-report questionnaires but they are likely to be more valid. There are no comparable data on the prevalence of binge eating among men or women outside the 1635 year age range.

Of the $3 \%$ or so of young women who binge at least weekly, about one-half have bulimia nervosa, a very small proportion have anorexia nervosa and most of the remainder fulfil the criteria for the new diagnosis of binge eating disorder.

\section{The management of bulimia nervosa}

In the relatively short time since bulimia nervosa was first described, much has been learned about its treatment: indeed, over the past decade bulimia nervosa has been one of the most intensively studied psychiatric disorders.

\section{In-patient treatment}

Both clinical experience and research evidence indicate that the great majority of patients may be managed on an out-patient basis. The few indications for admission are: depression of such severity that out-patient treatment is not possible, poor physical health (for example, severe electrolyte disturbance), and the failure of appropriate out-patient treatment.

When hospitalisation is necessary, it should always be viewed as a preliminary to out-patient care. In most cases the goal of hospitalisation should be simply to correct the problem that is preventing out-patient treatment, be it the patient's physical state or their level of depression. Once this has been done arrangements may be made for treatment proper to start on an out-patient basis. Clinicians should not be misled by the improvements that usually occur on admission to hospital: they merely reflect the influence of the hospital environment and rarely do they persist following discharge.

There is no consensus regarding the best way to manage those patients who are hospitalised as a result of the failure of appropriate out-patient treatment. Lacey (1995) advocates a multifaceted approach incorporating behavioural and psychodynamic elements, whereas Tuschen \& Bents (1995) have described an intensive cognitive-behavioural approach.

\section{Out-patient treatment}

\section{Antidepressant drugs}

The only pharmacological treatment to have shown promise is the use of antidepressant drugs. Controlled trials indicate that over a matter of weeks these drugs produce, on average, a $50-60 \%$ reduction in the frequency of binge eating, together with a substantial decrease in the level of associated psychiatric symptoms (see Wilson \& Fairburn, 1997, in press). No single antidepressant drug seems to be superior to any of the others, nor do the patients have to be depressed to benefit. Unfortunately, however, the changes generally do not last. For example, Walsh et al (1991) found that of the $41 \%$ of their sample who had responded sufficiently well to justify continuing taking the antidepressant drug desipramine, almost one-third $(29 \%)$ relapsed over the following three months. It is only a minority of these patients who 


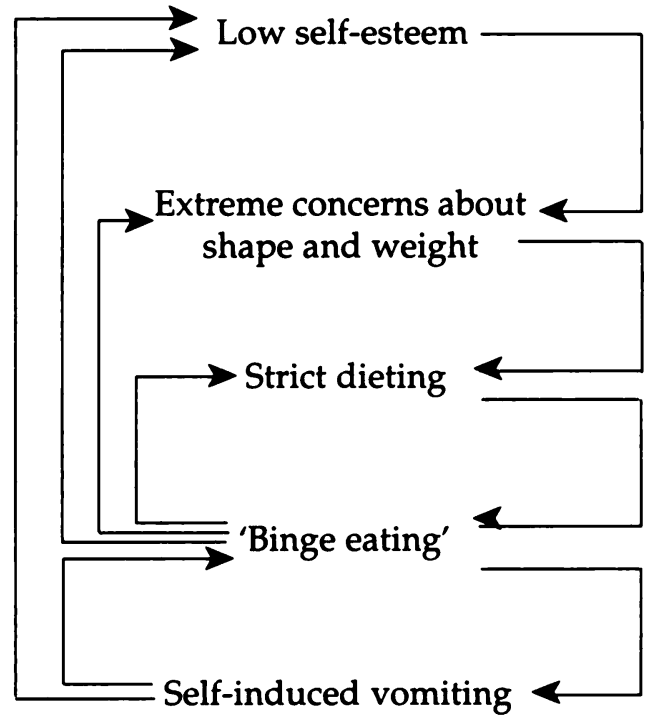

Fig. 2. The cognitive view of the maintenance of bulimia nervosa. Adapted with permission from Fairburn et al, 1993a.

obtain enduring benefit from antidepressant drugs. This is probably because the drugs fail to moderate the extreme and brittle dieting that is characteristic of bulimia nervosa and which is thought to maintain the binge eating.

\section{Cognitive-behavioural therapy}

The leading treatment for bulimia nervosa is a specific form of cognitive-behavioural therapy (CBT). This treatment is based on a cognitive view of the processes maintaining the disorder (Fairburn, 1997). According to this view (see Fig. 2) these patients' characteristically extreme concerns about shape and weight maintain the disorder through the influence of an extreme and rigid form of dieting. Various other vicious circles also operate, especially in those patients who attempt to compensate for their binges by vomiting or misusing laxatives. CBT aims to disrupt these maintaining mechanisms through the use of a specific sequence of behavioural and cognitive procedures. A detailed description of the treatment has been published (Fairburn et al, 1993a) and Box 1 lists its main elements. Generally, the treatment involves about 20 sessions over four to five months. No single professional group is inherently best qualified to administer the treatment; for example, psychiatrists, clinical psychologists and nurse therapists are all equally appropriate. Nor is the therapist's gender necessarily relevant. Some patients might be better treated by a female therapist; others might be better treated by a male therapist. What is of far greater importance than gender is the therapist's overall level of competence at administering CBT.
CBT has been the focus of over 20 controlled treatment trials, the results of which have consistently supported its use. Three main findings have emerged from these studies (Fairburn, 1996):

(1) CBT has a major beneficial effect on all aspects of the psychopathology of bulimia nervosa. There is a marked reduction (on average, about $80 \%$ ) in the frequency of binge eating and associated vomiting and laxative misuse, a decrease in dietary restraint and lessening in the intensity of the patients' concerns about shape and weight. Associated with these changes is a decrease in the level of general psychiatric symptoms and an improvement in self-esteem and social functioning.

Box 1 . The core elements of the cognitivebehavioural treatment for bulimia nervosa. (Adapted from Fairburn et al, (1993a).

Stage One

Presentation of the cognitive-behavioural view of the maintenance of bulimia nervosa

Establishing the self-monitoring of food and liquid intake and relevant cognitions

Education regarding body weight regulation, binge eating and the ineffectiveness of selfinduced vomiting and laxative misuse as methods of weight control

Introduction of a pattern of regular eating

Use of alternative behaviour to help resist urges to binge

Stage Two

Cognitive restructuring applied to concerns about food, eating, shape and weight, and, where relevant, other general cognitive distortions (e.g. perfectionism, negative self-evaluation)

Use of procedures to erode the various forms of dieting, including the systematic introduction of avoided foods

Development of problem-solving skills to apply to difficulties which might otherwise trigger binges

Use of behavioural and cognitive procedures to tackle body-image misperception and body-image disparagement

Stage Three

Use of procedures to minimise the risk of relapse, including education to ensure expectations are realistic and the formulation of a plan for use at times of difficulty 
(2) The changes obtained with CBT appear to be well maintained. Although there is a definite need for further studies on the medium- to longterm outcome following CBT, the available data suggest that the changes are robust. Between one-half and two-thirds have an excellent outcome, which is well maintained over the following six months to one year. The one study of longer-term outcome (mean (s.d.) length of follow-up 5.8 (2.0) years) found that two-thirds $(63 \%)$ of the patients had no eating disorder and the great majority were functioning extremely well (Fairburn et al, 1995).

(3) CBT has been found to be superior to all bar one of the treatments with which it has been compared. CBT has been compared with treatment with antidepressant drugs (and with antidepressant drugs and CBT combined), behavioural versions of CBT, exposure with response prevention, supportive expressive psychotherapy and interpersonal psychotherapy (IPT). It has been found to be superior to all these treatments, with the exception of IPT. Combining CBT and antidepressant drugs conveys no clear benefit.

\section{Interpersonal psychotherapy}

This is the one treatment that may be as effective as CBT. It is a short-term, focal psychotherapy developed by Klerman et al (1984) as a treatment for depression. The focus of the treatment is on helping patients identify and find solutions for current interpersonal problems. The one trial to have investigated IPT found that at the end of treatment it was less effective than CBT (Fairburn et al, 1991), but during follow-up the differences between the two treatments disappeared because of continuing improvement among the patients who received IPT (Fairburnet al, 1993b; 1995). Clinical experience suggests that IPT operates by improving interpersonal functioning, and thereby self-evaluation, which in turn results in a moderation of these patients' dependence upon shape and weight to evaluate their self-worth. As a result, weight control becomes of less importance to them, they diet less intensely and the eating disorder gradually erodes.

Before IPT can be recommended as an alternative to CBT it needs to be evaluated in further controlled trials. For the meantime, CBT must be regarded as the leading treatment for bulimia nervosa.

\section{Simplified cognitive-behavioural therapy}

The main obstacle to the widespread use of CBT is the amount of therapist time that it takes. Training is less of an issue since CBT is not an unduly difficult treatment to master.
There is growing evidence that a subgroup of patients responds to simplified forms of CBT and that these treatments need not be administered by therapists with special training (for review see Carter \& Fairburn, 1996). Of particular promise are the self-help books based on CBT. Two such books are available (Fairburn, 1995; Cooper, 1995), both of which are direct translations of the CBT for bulimia nervosa. Also, Schmidt \& Treasure (1993) have published a more eclectic programme, which includes some elements of CBT. These books may be administered either on their own (pure self-help) or with guidance from a non-specialist therapist (guided self-help), the latter approach usually involving six to eight 20- to 30-minute sessions over three to four months. Clearly, pure self-help has the potential to be eminently disseminable but, not surprisingly, some patients have difficulty persevering with it. In contrast, compliance is generally good with guided self-help. However, it must be noted that the current enthusiasm for these simplified versions of CBT is outstripping the evidence supporting their effectiveness. The few studies to date have had important methodological shortcomings, the most significant being that there has been little attempt to study maintenance of change. This is an important limitation given the tendency of binge eating problems to run a chronic course.

\section{Treatments for non-responders to CBT}

Cognitive-behavioural therapy is far from being a panacea. In research trials (generally using secondary or tertiary referrals) between one-half and two-thirds of patients obtain a substantial and lasting benefit, but the remainder respond either partially or not at all. Accordingly, there is a need to develop other methods of treatment.

Wilson (1996) has reviewed the main alternatives to CBT. Antidepressant drugs and IPT are obvious options since both are established treatments in their own right. However, there is no a priori reason why they should benefit those who do not respond to CBT. Other alternatives include psychodynamic psychotherapy and family therapy. Once again, there is no reason to think that these treatments will succeed where CBT has failed. There has been just one controlled study of a psychodynamic treatment, supportive-expressive psychotherapy, and the results indicated that it was less effective than CBT (Garner et al, 1993). Similarly, a study of family therapy obtained disappointing results (Russel et al, 1987). In support of psychodynamic psychotherapy it is sometimes argued that it is particularly well suited to the treatment of patients with a co-existing personality disorder. Although it is certainly true that 
patients with severe personality disorders are overrepresented among CBT non-responders, there is no evidence to suggest that psychodynamic psychotherapy is any more effective with this particular patient group.

Rather than replacing CBT with a different form of treatment, it is the author's view that there might be more to be gained by adapting CBT. Several possibilities suggest themselves. One would be to broaden the focus to address general issues such as self-esteem and interpersonal functioning. However, as Wilson (1996) points out, there is no evidence that 'broad' CBT is superior to a more focused approach, either with respect to this disorder or any other. Another possibility would be to incorporate features of Linehan's CBT for borderline personality disorder (Linehan, 1993). A third possibility would be to intensify the treatment in such a way as to ensure that compliance with key procedures is enhanced. It is common clinical experience that many $\mathrm{CBT}$ non-responders have either failed to follow key assignments or have followed them to a limited extent. If their compliance were to be enhanced, the outcome might be improved. With this in mind, the cognitivebehavioural in-patient programme of Tuschen \& Bents (1995) might have something to offer, especially since it could be adapted for use on a day patient or out-patient basis. Research on the best way to manage CBT non-responders is urgently needed.

\section{The treatment of other binge eating disorders}

\section{Anorexia nervosa}

In comparison with the large body of research on the treatment of bulimia nervosa, there has been little work on the treatment of the other disorders in which binge eating features. For example, comparatively little has been written on the management of those patients with anorexia nervosa who binge. (The management of anorexia nervosa was discussed recently in this journal; Palmer, 1996.) In the author's experience the best approach is to treat these patients in the same way as other anorectics, giving due priority to the need for weight gain. Once the patient's weight has been restored to a reasonable level, or earlier if need be, the binge eating may be addressed using procedures from CBT for bulimia nervosa.

\section{Binge eating disorder}

This disorder is attracting considerably more attention, particularly when it co-occurs with obesity. Not surprisingly, investigators are showing most interest in testing those treatments that have been shown to benefit patients with bulimia nervosa. Thus antidepressant drugs, CBT, IPT and cognitive-behavioural self-help have all been evaluated (Wilson \& Fairburn, 1997, in press). As in the treatment of bulimia nervosa, relapse appears to be a problem with the use of antidepressant drugs. The outcome with CBT and IPT also seems disappointing, possibly because the studies have administered the treatment in a group rather than individual format. Much better results are being reported from a study from Pittsburgh in which CBT and a behavioural weight control programme were compared, both treatments being administered on a one-to-one basis. Substantial and sustained changes were obtained with both interventions and in the case of the behavioural weight control programme there was also significant weight loss. If this finding is confirmed, it would suggest that dietary/behavioural techniques alone may be sufficient to treat binge eating disorder, whereas their use is associated with relapse in bulimia nervosa. It is also of note that preliminary data from a trial evaluating cognitivebehavioural self-help suggest that a simple intervention of this type may also benefit some patients with binge eating disorder.

\section{The notion of 'stepped care'}

How can these research findings be implemented in routine clinical practice? It has been suggested that a 'stepped care' approach would be a logical way of managing many of these patients. In this approach, patients would be treated first with a simple treatment, and would move on to a more complex one only if they failed to respond. Two possibilities suggest themselves for the first step; either pure self-help or guided self-help. The research to date suggests that a worthwhile proportion would respond to one or other approach, although the stability of the response has not been established. Those who do not benefit from this initial intervention, or who make only temporary gains, would move on to the second step. In this case, full CBT would seem the most appropriate option. The choice of treatment for the third step is far from clear, given the uncertainty 
over how best to help CBT non-responders. At this point clinicians would need to rely on a trial-anderror approach.

\section{References}

Carter, J. C. \& Fairburn, C. G. (1995) Treating binge eating problems in primary care. Addictive Behaviours, 20,765-772.

Cooper, P. J. (1995) Bulimia Nervosa and Binge-Eating: A Guide to Recovery. London: Robinson.

Fairburn, C. G. (1995) Overcoming Binge Eating. New York: Guilford Press.

- (1997) Eating disorders. In Science and Practice of Cognitive Behaviour Therapy (eds D. M. Clark \& C. G. Fairburn), pp. 209-241. Oxford: Oxford University Press.

- \& Beglin, S. J. (1990) The studies of the epidemiology of bulimia nervosa. American Journal of Psychiatry, 147, 401-408.

- Jones, R., Peveler, R. C., et al (1991) Three psychological elements for bulimia nervosa: A comparative trial. Archives of General Psychiatry, 48, 463-469.

---, Marcus, M. D. \& Wilson, G. T. (1993a) Cognitivebehavioural therapy for binge eating and bulimia nervosa: A comprehensive treatment manual. In Binge Eating: Nature, Assessment and Treatment (eds C. G. Fairburn \& G. T. Wilson), pp. 631-404. New York: Guilford Press.

-, Jones, R., Peveler, R. C., et al (1993b) Psychotherapy and bulimia nervosa: The long-term effects of interpersonal psychotherapy, behaviour therapy and cognitive behaviour therapy. Archives of General Psychiatry, 50, 419-428.

-, Norman, P. A., Welch, S. L., et al (1995) A prospective study of outcome in bulimia nervosa and long-term effects of three psychological treatments. Archives of General Psychiatry, 52, 304-312.

Garner, D. M., Rockert, W., Davis, R., et al (1993) Comparison of cognitive-behavioural and supportive-expressive therapy for bulimia nervosa. American Journal of Psychiatry, 150, 37-46.

Klerman, G. L., Weissman, M. M., Rounsaville, B. J., et al (1984) Interpersonal Psychotherapy of Depression. New York: Basic Books.

Lacey, J. H. (1995) Inpatient treatment of multi-impulsive bulimia nervosa. In Eating Disorders and Obesity: $A$ Comprehensive Handbook (eds K. D. Brownell \& C. G. Fairburn), pp. 361-368. New York: Guilford Press.

Linehan, M. M. (1993) Cognitive-Behavioural Treatment of Borderline Personality Disorder. New York: Guilford Press.

Palmer, R. L. (1996) The management of anorexia nervosa. Advances in Psychiatric Treatment, 2, 61-68.

Russel, G. F. M., Szmukler, G. I., Dare, F., et al (1987) An evaluation of family therapy in the treatment of anorexia nervosa and bulimia nervosa. Archives of General Psychiatry, 44, 1047-1056.

Schmidt, U. \& Treasure, J. (1993) Getting Better Bit(e) by Bit(e). Hove: Lawrence Erlbaum.

Tuschen, B. \& Bents, H. (1995) Intensive brief inpatient treatment of bulimia nervosa. In Eating Disorders and Obesity: AComprehensive Handbook (eds K. D. Brownell \& C. G. Fairburn), pp. 354-360. New York: Guilford Press.

Walsh, B. T., Hadigan, C. M., Devlin, M. J., et al (1991) Longterm outcome of antidepressant treatment for bulimia nervosa. American Journal of Psychiatry, 148, 1206-1212.

Wilson, G. T. (1993) Assessment of binge-eating. In Binge Eating: Nature, Assessment and Treatment (eds C. G. Fairburn \& G. T. Wilson), pp. 227-249. New York: Guilford Press.

- (1996) Treatment of bulimia nervosa: When CBT fails. Behaviour Research and Therapy, 34, 197-212.

- \& Fairburn, C. G. (1997) Eating disorders. In Psychotherapies and Drugs that Work: A Review of Outcome Studies (eds P. E. Nathan \& J. M. Gorman). Oxford: Oxford University Press, in press.

\section{Multiple choice questions}

1. Binge eating is associated with:
a anorexia nervosa
b bulimia nervosa
c pica
d binge eating disorder
e atypical eating disorders.

2. The studies of the treatment of bulimia nervosa have shown the following:

a antidepressant drugs have a role only if the patient is depressed

b appetite suppressants decrease the urge to binge

c family therapy shows promise

d CBT is associated with relapse

e interpersonal psychotherapy may have a role.

3. CBT for bulimia nervosa:

$\mathrm{a}$ is best conducted on an in-patient basis

b takes about five months

c aims to strengthen the patients' ability to diet

d works faster than interpersonal

psychotherapy

e female therapists are to be preferred.

4. The following treatments have been proposed for non-responders to CBT:
a hypnosis
b antidepressant drugs
c in-patient treatment
d jaw wiring
e interpersonal psychotherapy.

5. In binge eating disorder:

a weight restoration is an important goal

b dietary/behavioural treatments make the binge eating worse

c appetite suppressants show promise

d group CBT has been evaluated

e obesity may be an associated problem.

\begin{tabular}{|c|c|c|c|c|}
\hline \multicolumn{5}{|c|}{ MCQ answers } \\
\hline 1 & 2 & 3 & 4 & 5 \\
\hline a $T$ & a $F$ & a $F$ & a $F$ & a $F$ \\
\hline b $T$ & b F & b $T$ & b $T$ & b $F$ \\
\hline c $F$ & c $\mathbf{F}$ & c $\mathrm{F}$ & c $\mathrm{T}$ & c $\mathrm{F}$ \\
\hline d $\mathrm{T}$ & d F & d $\mathrm{T}$ & d F & d $\mathrm{T}$ \\
\hline e $\mathrm{T}$ & e $\mathrm{T}$ & e $F$ & e $\mathrm{T}$ & e $\mathrm{T}$ \\
\hline
\end{tabular}

